\title{
The status of Big Bang nucleosynthesis in July 1988
}

France

Hubert Reeves Section d'Astrophysique C.E.N.S. Saclay Gif F - 91191

Institut d'Astrophysique de Paris 75014 Paris

years.

Important progresses have been made in two fronts in the few past

On the theoretical ground, we have realized the importance of the quark-hadron phase transition as possibly influencing in a major way the yields of the primordial nucleosynthesis isotopes.

On the observational ground, the status of lithium as a bona-fide cosmological observable has been confirmed and its primordial abundance can be evaluated with increased confidence.

\section{The quark-hadron phase transition.}

The physics of the quark-hadron phase transition (or transitions, since there is a chiral transition and a confinement transition) is presently the object of intense studies (Iso et al 1986) (Satz 1985,1987) (Leutwyler, 1988) etc). Many of the parameters of the transitions are still poorly known despite the vigourous effort being made in QCD calculations on networks.

The relevant parameters, as far as BBN is concerned, are the following. First: the order of the transitions. There are strong indications that in the baryonic density range of the $\mathrm{BBN}$, the transitions are first order, leading to nucleation and to bubbles of high density matter in a low density background ( or the inverse).

Second : the critical temperature $T_{c}$ of the transitions. Again in the density range of cosmological interest, the transitions appear to occur at the same $T_{c}$ and to be simultaneous. QCD calculations give a range of $150 \mathrm{MeV}<\mathrm{T}_{\mathrm{c}}<250 \mathrm{MeV}$. Recent chiral perturbation calculations have been published which quote a narrower range of $180 \mathrm{MeV}<\mathrm{T}_{\mathrm{c}}<220 \mathrm{MeV}$ (Gasser and Leutwyler, 1987, 1988)

The density contrast $R$ between the high and low baryonic density phases can be computed, assuming chemical potential equilibrium. between the two phases (Sale and Matthews 1986) (Applegate and Hogan 1985) (Applegate, Hogan and Sherrer 1987), (Alcock et al 1987) (Fuller et al 1987) (Kapusta and Olive 1988). The result depends strongly on the value of the assumed critical temperature. At low $T_{c}$, the computed value of $R$ is larger than ten, decreasing gradually at higher $T_{c}$. Thus, through the value of $R$, the lithium abundance is related to the value of $T_{c}$. In reality the gradual hadronization of the quark sea leads to a distribution of baryonic densities.

As the universe cools from $\mathrm{T}_{\mathrm{c}}$, at approximately $20 \mu \mathrm{sec}$, to one $\mathrm{MeV}$, at one second, the contrast $R$ between the phases is maintained. The neutron to proton ratio $(\mathrm{n} / \mathrm{p})$, governed by weak processses, is the same in all phases. It is given by the 
Boltzmann formula of mass-action. Below one $\mathrm{MeV}$ the weak processes are no more in thermal equilibrium. The neutrons diffuse from high density phases into low density phases, changing both their density and their $n / p$ ratio.

It may be a long time before we get definite results on the effect of the quark-hadron phase transitions on the formation rate of the cosmological nuclides. Nevertheless recents calculations, especially those taking into account the effect of neutron back-diffusion during nucleosynthesis ( Terasawa and Sato 1988, KurkiSuonio et al (1988) already give us the general trends.

We may expect the final results to be quite comparable with the results of homogeneous density calculations. Our present ignorance of the exact values of many relevant parameters of the $\mathrm{Q}-\mathrm{H}$ transition can be assimilated to corresponding uncertainties on the final results. These uncertainties are likely to decrease as more detailed studies of the transition become available.

Taking into account these various effects and the associated uncertainties in the parameters of the transitions, various calculations have been made of the primordial nucleosynthesis yields of the cosmological isotopes. The present state of the art can be summarized in the following sentences.

Given the all the uncertainties, including those related to the determination of the Hubble parameter, the range of $\Omega \mathrm{b}$ goes from 0.1 to 0.01 . This is appreciably larger than in the case of a homogeneous density universe. This does not appear to be large enough to allow the baryons to close the universe $\left(\Omega_{b}<1\right)$.

The cosmic density of luminous matter (stars and X-ray cluster gas) is $\Omega_{\mathrm{L}}=$ 0.01 whitin a factor of two ,while the density of clustered matter needed to account for the stability of clusters of galaxy or large scale motions is $\Omega_{\mathrm{G}}=0.1$ to 0.2 .

Thus, within the uncertainties, at one end of the scale the baryonic matter could be entirely luminous (no baryonic dark matter) while at the other end of the scale the clustered matter could be entirely baryonic ( no non-baryonic dark matter).

The comparison between the present calculations and the cosmic abundances suggest that the contrast $R$ between the phases is unlikely to be larger than ten. This result is in agreement with the best estimate of the critical temperature of the phase transition ( $180 \mathrm{MeV}<\mathrm{T}_{\mathrm{c}}<220 \mathrm{MeV}$ ) leading to $\mathrm{R} \approx 7$.

\section{The promotion of ${ }^{7} \mathrm{Li}$ to the status of "cosmological observable".}

Thanks to a number of new developpments, both observational and theoretical, the isotope lithium-7 has gradually been promoted to the status of a bona fide cosmological observable. First because its BBN contribution can now be evaluated more properly. Second because its potential message has been clarified, in relation with baryon inhomogeneities (as stressed, for example, by the anaysis of the physics of the quark-hadron phase transition. (Witten 1984)).

One of the most important event in observational cosmology in recent years has been the discovery of lithium in PopII stars by the Spite ( $1983 \mathrm{a}$ and $\mathrm{b}$ ). Later, a number of other observations have confirmed their data and added a wealth of new measurements (Spite et al 1985) (Hobbs and Duncan 1987) (Rebolo, Molaro and Beckman 1987). The full data and its significance are best presented as a function of the hydrogen to iron ratio. This parameter is a measure of the importance of stellar nucleosynthesis on a galactic scale The abundance of lithium has remained almost constant (within a factor of two) while iron grows from .0003 to 0.1 of the solar value. The message is clear : the lithium in this range is not mostly produced by stellar 
processes. There exist a primordial component which dominates the stellar contribution all through this range.

Observations have shown that this component is mostly made of ${ }^{7} \mathrm{Li}:\left({ }^{7} \mathrm{Li} / 6 \mathrm{Li}\right.$ $>10$, Maurice et al 1984). This, however, is not a very tell-taling result since in typical stellar outer layers $6 \mathrm{Li}$ is thermonuclearly destroyed one hundred times faster than $7_{\mathrm{Li}}$.

More interesting results come from beryllium and boron, two elements which are produced in Galactic Cosmic Rays ( Meneguzzi et al 1971) but not in BBN ( Wagoner et al 1967). The rate of formation of lithium (both isotopes) is, to better than a factor of two, the same as the rate of formation of boron (both isotopes). It is approximately ten times larger than the rate of formation of beryllium ( Reeves and Meyer, 1978, Walker et al 1985). Furthermore, lithium, at all relevant temperature, is destroyed faster than beryllium and boron by stellar processes. Thus the abundance of beryllium gives an estimate of the GCR contributed lithium in a star while the abundance of the boron gives an upper limit to the GCR contribution.

Beryllium has been recently detected in Pop II stars (Rebolo, Molaro, and Beckman 1988) with hydrogen ratio $9 \mathrm{Be} / \mathrm{H}=2 \times 10^{-12}$. The corresponding GCR lithium is only one tenth of the observed Pop II abundance.

A search for boron ( Molaro 1987) in a Pop II star (HD 140283) has yielded an upper limit of $\mathrm{B} / \mathrm{Li}<0.04$. The corresponding upper limit to the $7_{\mathrm{Li}}$ isotope is $(7 / \mathrm{H}) \mathrm{GCR}<10^{-11}$, at least an order of magnitude smaller than the PopII observations Thus we may conclude that the primordial lithium component is not the result of hypothetical primordial cosmic rays (Montmerle 1977). The only other process known to us to generate lithium-7 in interesting amount is BBN and we may thus conclude that the lithium in Pop II stars mostly is of cosmological origin.

But, in order to recover the primordial abundance relevant to BBN model calculations, we must face the question of possible depletion of this element by stellar surface processes.

The mean abundance of lithium in Pop II stars is $7 \mathrm{Li} / \mathrm{H}=1.6 \times 10-10$ with a dispersion of a factor of two (Rebolo et al 1987). This small dispersion is the main argument in favor of the hypothesis that this lithium abundance has not suffered much depletion by processes associated with the stellar surfaces ( Michaud 1986). Following the same logic, it appears reasonable to estimate that the fractional depletion should not be larger as the observed dispersion. A thorough study of surface depletion processes by Delyannis et al (1988)has given a similar result . In consequence we estimate an initial value of $7 \mathrm{Li} / \mathrm{H}=2.0+0.5 \times 10^{-10}$.

Vauclair $(1987,1988)$, studying the effect of rotational mixing on stellar lithium, has argued that the depletion may have been larger and that the primordial value may be the same as the Pop 1 value $\left(7_{\mathrm{Li}} / \mathrm{H}=1.0 \times 10^{-9}\right)$. It remains to be seen if the theory will be able to reproduce the small abundance dispersion displayed in figure 1. More work is being done on this subject.

\section{Bibliography}

Alcock, C.R., Fuller, G.M., and Mathews, G.J., Astrophys. J. 320, 439, 1987

Applegate, J.H., and Hogan, C. 1985 Phys . Rev. D31 3037.

Applegate. J.H., Hogan, C., and Sherrer R.J. 1987a Phys Rev. , D35 , 1151.

Cayrel. R., I.A.U Symposium no 126 Cambridge 1986.

Cayrel . R., Proceedings of the Alpbach Summer school 1988.

Delyannis, C., Demarque, P., Kawaler, S., Krauss, L., and Romanelli, P. preprint, (1988).

Fuller ,G.M., Mathews G.J., and Alcock, C.R. Phys. ReV . D37 13801988 
Gasser , J and Leutwyler, H., Light quarks at low temperature. Phys Lett B 184, 83 , 1988.

Gasser , J and Leutwyler, H., Thermodynamics of chiral symmetry. Phys Lett B 188 , $477,1987$.

Hobbs, L.M., and Duncan, D.K., Ap.J. 317, 796, 1987. ( Li in halo stars)

Iso, K., Kodama, H., and Sato, K., Phys. Lett. 337, 169B, 1986.

Kapusta,J.I., and Olive K.A. 1988 preprint

Kurki-Suonio, H., Matzner , M.M., Centrella, J.M., Rothman, T., and Wilson, J.R., , preprint submitted to Physical Review D 1988 preprint

Leutwyler, H., QCD; low temperature expansion and finite -size effects. Proceedings of the Seillac Conference to appear in April 88.

Maurice, E., Spite., F., and Spite , M., Astron. Astrophys132, 278 , 1984 ( Li-7 / Li -6 ratio in old stars.)

Meneguzzi,M., Audouze, J., and Reeves , H., 1971, Astr. Ap., 15, 337.

Michaud, G., Ap. J. 302, 650, 1986

Molaro. P. Astr. Ap. 183,394 1987 (boron in Pop II)

Montmerle 1977 thesis Université de Paris.

Rebolo, R., Beckman,.J., and Molaro, P., Astron, Astrophys. 172 L17 1987 ( Li in G $64-12$ )

Rebolo, R., Molaro, P., Abia, C., and Beckman, J.E., Astron. Astrophys., 193, 193-201, 1988. ( Be-9 in Pop II)

Reeves, 1971, p. 256, American Physical Society Meeting, Porto Rico Dec 1971

Reeves . H., Ann Rev Astron Astrophys. 12,437, 1974

Reeves, H. Varenna School "Confrontations between Observations and Theories in Cosmology" July 1987

Reeves, H., Delbourgo-Salvador, P , Audouze, J. , and Salatti, P. to appear in European Journal of Physics august 88

Reeves, H., and Meyer, J.P., 1978, Astrophys. J. 226, 613.

Reeves, H., and Richer, J., 1988 in preparation.

Sale, K.E. and Mathews, G.J. 1986 Ap.J. 309 L1

Satz ,H., Ann. Rev. Nucl. Sci. 351985

Satz,H., Proceedings of the Strasbourg Symposium on the quark-hadron phase transition. July 1987

Spite, M and Spite, F. 1983a Nature 297, 483.

Spite, F. and Spite. M. 1983b Astr. Ap ., 115, 337.

Spite, F., Spite, M., Peterson, R.C., and Chafee, F.H., Astron. Astrophys. 172 L9 1987, ( $\mathrm{Li}$ in metal poor halo stars).

Spite F. and M., $1985\left({ }^{7} \mathrm{Li} / 6 \mathrm{Li}>10\right)$

Terasawa, N., and Sato, K., preprint (1988)

Vauclair, S., Procedings of Meudon IAU Symposium July 1987

Vauclair, S., 1988 preprint.

Walker, T.P., Mathews, G.J. and Viola, V.E. 1985, Ap.J., 299, 745.

Wagoner, R. V., Fowler, W. A., and Hoyle, F., Ap.J. 148, 3, 1967.

Witten E., 1984 Phys. Rev. D30 272

Yang , J., Turner, M.S., Steigman, G., Schramm, D.N., and Olive, K, 1984, Ap.J. , 281, 493 